\title{
De nouveaux modes de contestation dans le monde arabe
}

Bernard Botiveau

\section{(2) OpenEdition}

1 Journals

\section{Édition électronique}

URL : http://journals.openedition.org/conflits/139

DOI : $10.4000 /$ conflits.139

ISSN : $1777-5345$

Éditeur :

CCLS - Centre d'études sur les conflits lilberté et sécurité, L'Harmattan

\section{Édition imprimée}

Date de publication : 21 mai 1992

ISSN : 1157-996X

Référence électronique

Bernard Botiveau, « De nouveaux modes de contestation dans le monde arabe », Cultures \& Conflits [En ligne], 05 | printemps 1992, mis en ligne le 13 mars 2006, consulté le 30 mars 2021. URL : http:// journals.openedition.org/conflits/139; DOI : https://doi.org/10.4000/conflits.139

Ce document a été généré automatiquement le 30 mars 2021.

Creative Commons License 


\title{
De nouveaux modes de contestation dans le monde arabe
}

\author{
Bernard Botiveau
}

La visite officielle effectuée le 13 décembre 1991 à Khartoum par le président iranien Rafsandjani, l'inquiétude manifestée par les gouvernements du Maghreb devant les contacts opérés au cours des derniers mois de 1991 entre dirigeants soudanais et militants islamistes maghrébins, la poussée électorale du FIS le 26 décembre 1991 en Algérie : ces faits récents conduisent à examiner l'hypothèse d'une recomposition de la contestation politique dans le monde arabe et musulman, après la guerre du Golfe. Peut-on décrire une nouvelle carte de la contestation au sein de "sociétés du Sud" où une nouvelle génération d'acteurs politiques puiserait dans une idéologie islamiste universalisable les moyens d'une mobilisation des ressources symboliques de la lutte politique contre des gouvernements en mal de légitimité ? En mettant l'accent de façon trop unilatérale sur la puissance mobilisatrice d'un islam politique varié, on se prive probablement de dimensions explicatives propres aux sociétés arabes. Quoique prises depuis la guerre du Golfe dans des stratégies internationales qui débordent les cadres de référence élaborés par le nationalisme arabe, ces sociétés restent liées par une communauté d'intérêts en même temps que par la mémoire de divergences, pôles entre lesquels ont régulièrement oscillé, depuis près d'un demi-siècle, des aspirations unitaires et des tendances centrifuges. Concernant ces dernières, il faut d'abord rappeler, au plan national, la diversité des états constitués au lendemain d'indépendances qui ne furent pas simultanées: états confrontés aux exigences du développement, à des territorialisations en partie héritées de la colonisation, et devant composer avec des clivages sociaux et culturels particuliers. Ces spécificités ont contribué à alimenter les débats sur l'unité où était mise en jeu la question de l'identité arabe. Quant aux processus unitaires, ils ont été maintes fois malmenés sur le plan international par des tentatives extérieures de division ${ }^{1}$, par des incompatibilités internes entre systèmes de légitimation politique, quand ce ne fut pas par l'éloignement géographique de plusieurs ensembles pris dans leurs allégeances externes respectives. Par exemple le Maghreb resta plus ou moins éloigné des débats politiques du Machreq, même s'il fut toujours concerné par la question palestinienne 
dont la formulation actuelle de la contestation, l'Intifada, s'est fortement inscrite dans l'imaginaire de ses sociétés ${ }^{2}$. En dépit de ces réserves, il est permis de s'interroger sur la capacité de mouvements radicaux qui, constatant l'échec de modèles antérieurs érodés, cherchèrent à élaborer un modèle unitaire valable pour le monde arabe et plus largement pour le monde musulman; modèle qui correspondrait, en quelque sorte, à un second souffle du nationalisme arabe. En effet, sans se livrer ici à une aléatoire typologie des processus antérieurs de mobilisation dans le monde arabe, il est malgré tout utile de situer ce qui, dans l'histoire de ces dernières décennies, a représenté des moments importants de mobilisation sociale dans un contexte marqué au sceau de l'anti-impérialisme (moment des mouvements nationaux puis des partis nationalistes, moment ensuite de l'instabilité chronique et relative générée par les politiques d'ouverture des années 70 et alimentée en permanence par le non-règlement de la question palestinienne) afin de comprendre le lien qui les relie à la recherche d'une identité actuellement définie par les bases d'un islam politique réécrit par l'ijtihâd politique des islamistes (en particulier chiites et sunnites). La récente guerre du Golfe a relancé ces mouvements de contestation dont certaines valeurs communes (défense de l'arabisme et des lieux saints de l'islam, défense de la cause palestinienne à travers son nouveau "champion", Saddam Hussein) ont nourri, sur le terrain, des stratégies spécifiques, car tributaires localement des choix politiques faits par les états face à l'occupation du Koweït par l'Irak. Cependant, si la guerre a accru l'identification islamique de ces mouvements, tout en renforçant leurs racines nationales, leurs divergences témoignent aussi de leur interaction complexe avec les facteurs anciens. Les exemples choisis ici devraient aider à montrer comme un référent islamique relativement homogène s'est alors inséré dans l'argumentaire des nationalistes arabes, avant d'être monopolisé par des mouvements islamistes arabes dont le dernier en date est l'islamisme algérien.

2 L'héritage nationaliste Comment s'articulent aujourd'hui la conscience d'un espace hérité et le souvenir de moments fondateurs réinterprétés à la lecture d'une modernité définie ailleurs et imposée par tous ses stratagèmes, à commencer par la violence : cette question reste certainement très présente dans les représentations politiques actuelles. Trois moments importants de mobilisation jalonnent depuis le début de ce siècle la structuration et l'affaiblissement du nationalisme arabe au rythme des mutations de l'ordre international guerres mondiales ou paix armées. Les débuts du panarabisme Tout d'abord, après la Première Guerre mondiale, un panarabisme, qui s'est forgé contre le pouvoir ottoman, s'investit dans les "pays de Syrie" contre les deux bénéficiaires principaux de la guerre, l'Angleterre et la France. Après une brève expérience d'autonomie, la Syrie se mobilisera contre le Mandat, à travers la révolte druze de 1925 ou les manifestations urbaines orchestrées par le Bloc national ; l'égypte choisira quant à elle une voie originale à partir de sa révolution de 1919 et ses révoltes citadines, et l'Irak comptera sur la résistance de ses oulémas chiites à l'affirmation d'un pouvoir sunnite surveillé par l'Angleterre. L'anticolonialisme de cette époque avec son expérience limitée de systèmes parlementaires inédits, demeure présent dans la mémoire politique des sociétés arabes du Machreq, malgré le discrédit jeté ultérieurement sur certains de ses acteurs par les nationalistes arabes.

3 Les contestations à la sortie de la décolonisation Cette identification dans la contestation culminera après 1945, avec des promesses de décolonisation non tenues, attisée par les difficultés économiques de populations très durement éprouvées par la 
guerre, puis par les débuts de la guerre froide et surtout par la création de l'état d'Israël en 1948. La révolte éclate à Sétif en 1946, en égypte en 1946, en 1948 et surtout en janvier 1952, avec l'incendie de quartiers du Caire perçus comme les lieux privilégiés de la présence coloniale. En Syrie, elle sera canalisée en 1949 par des pronunciamientos successifs à commencer par celui d'inspiration kémaliste, de Ilusni Al-Za'îm. Au cours de cette période, la contestation au nom de l'indépendance et de l'unité nationales favorise la coexistence, quand ce n'est pas le regroupement de courants politiques aussi différents en égypte que le wafdisme, les Frères musulmans, le libéralisme constitutionnel ou le mouvement Jeune égypte.

4 L'installation à l'imitation de l'égypte de régimes nationalistes producteurs de gestes symboliques, comme le fut la nationalisation du Canal de Suez en 1956, s'accompagne de manifestations de rue, auxquelles la puissante "Voix des Arabes" n'est pas vraiment étrangère. Une imposante mobilisation populaire opère alors au nom d'idéaux qui s'avéreront inégalement profitables aux classes sociales des pays concernés; elle se fait en dénégation de systèmes politiques vieillis et dans une dynamique unitaire; tel fut le cas en 1958 avec le soutien initialement massif accordé par la population syrienne à la RAU, qui précède de quelques années le triomphe des formations baasistes. Dans ces nationalismes et avec les variantes nassérienne et baasiste, la culture islamique est saisie comme un élément de la personnalité nationale parfaitement intégrable dans la consolidation d'états-nations s'orientant vers un système socialiste ${ }^{3}$. Le Maghreb est attentif à ces processus de libération nationale dans le cadre de ses luttes pour l'indépendance.

5 Les exigences culturelles et politiques L'espoir d'un accès à une liberté politique définie dans un cadre national maîtrisé par le pouvoir, commencera à se lézarder dans les années 70 lorsque la Syrie, puis l'égypte - respectivement à partir de 1970 et 1974 seront contraintes de pratiquer non seulement des ouvertures économiques mais aussi une ouverture vers plus de libertés car l'absence de ces dernières était rendue insupportable par les difficultés nouvelles: pression démographique et endettement croissant. Ces années ont alors amorcé une phase différente au cours de laquelle l'abandon d'une unité de façade a rendu beaucoup plus visible des antagonismes irréductibles, comme en égypte où Sadate tenta de "jouer" des mouvements islamistes naissants contre une gauche marxiste et nassérienne, méfiante à l'égard du libéralisme économique de l'Infitâh et fidèle à ses engagements antisionistes. Si les émeutes du pain, qui deviendront des "émeutes FMI", traduisent avant tout, à la fin des années 70 et au cours des années 80 (1977 en égypte, 1978 en Tunisie, 1984 en Tunisie et au Maroc notamment), une demande de mieux-être, elles sont aussi traversées de toutes les contradictions qui nourriront des projets "islamiques" de réorganisation sociale, sanctionnant une délégitimation, déjà bien perçue, des régimes politiques en place ${ }^{4}$. $\mathrm{Ce}$ rappel, même bref, de quelques processus de mobilisation bien connus vise à souligner l'interaction des explications possibles de ces changements de légitimité : explication d'ordre socio-économique, recours à la menace extérieure, dimension culturaloreligieuse de la contestation. La décennie 80 fut certainement marquée par une lecture de ces processus où les acteurs politiques "légaux" ont été amenés à s'orienter, sur la base d'un constat d'échec, vers des explications d'ordre culturel, reconnaissant par là même la capacité des mouvements islamistes à se faire investir d'une légitimité nouvelle; car ils expriment au plus près les aspirations de la masse des déshérités, au point que le terme de déshérités (mustadafîn), popularisé par la révolution iranienne, désignera l'objet même du combat dans un langage à connotation religieuse ${ }^{5}$. Ce fait 
n'est pas en soi surprenant, quand on sait la centralité des valeurs islamiques dans la société : en égypte en 1882, le mouvement orabiste mobilisait ses troupes en invoquant le djihâd, comme le fera quelques années après le jeune Parti national et plus tard les révolutionnaires algériens du $\mathrm{FLN}^{6}$. Le parti Jeune égypte, créé en 1939, était partisan d'une application de la sharî'a élément du patrimoine arabe, sans pour autant envisager une "islamisation" de la société. Ce qui est probablement nouveau c'est que ce langage est à présent intériorisé par un nombre croissant d'acteurs, notamment parmi les intellectuels, comme le montreront les exemples qui suivent. Mais cet élément religieux n'est pas le seul à prendre en compte. De même que l'on peut expliquer la carence démocratique actuelle de nombre de sociétés arabes par un faisceau de facteurs structurels et conjoncturels ${ }^{7}$, il est permis de s'interroger sur la permanence et la lourdeur de facteurs, qui, comme c'est le cas pour la non-satisfaction d'aspiration démocratiques, "produisent" de la contestation; et cela, sans suivre pour autant les analyses portant sur une "culture de l'émeut, qui ne serait que le dernier recours pour supporter l'insupportable (puisque tout changement en profondeur serait interdit par le "mode de production dominant") $)^{8}$. "L'islamisation" de la contestation : trois exemples 1973 La Syrie contre la Constitution baasiste L'année 1973 qui culmina en octobre avec l'offensive syro-égyptienne contre Israël fut marquée durant les mois de février et de mars par de graves manifestations urbaines, dont l'enjeu était l'adoption d'une nouvelle Constitution, Constitution qui devait être adoptée le 8 mars, jour du 10ème anniversaire de la révolution baasiste ${ }^{10}$. Or ce qui devait apparaître comme un plébiscite du président Hafez Al-Asad, au pouvoir depuis 1970, se transforma en une confrontation ouverte avec une opposition regroupée autour des oulémas syriens. Dans un premier temps, les grands oulémas, soutenus par des manifestations de rue à Hama, appelèrent au boycott du référendum si l'omission dans le projet de Constitution d'un article affirmant que le chef de l'état est musulman n'était pas corrigée. Contrairement à la plupart des pays arabes, ce texte ne semblait pas s'imposer en Syrie, dans la mesure où aucune Constitution depuis l'indépendance n'avait contenu cet article, et que, de plus, l'islam étant selon l'idéologie baasiste une des composantes fondamentales de l'arabité, la référence religieuse était considérée comme implicite. Néanmoins, après des affirmations officielles selon lesquelles le projet était "l'expression de la voie droite" ${ }^{11}$, le gouvernement recula et intégra l'article exigé dans le projet. L'opposition ne désarma pas, exigeant davantage encore, mais cette fois par la voix d'oulémas de rang moins important, qui relancèrent la contestation dans les villes du centre de la Syrie et même à Damas. Un nouvel article, citant le fiqh (jurisprudence islamique) comme une "source principale de la législation" fut introduit dans le projet, tandis que la rébellion était réprimée par la force et le texte définitif de la Constitution adopté, avec quelques jours de retard. Cette contestation de caractère clairement islamique doit cependant être replacée dans le contexte de la Syrie des années 70. Au cours des premières années de la révolution baasiste (1963-1966), l'accent mis sur le nationalisme arabe, puis sur le socialisme ne pouvait masquer un changement des allégeances : après avoir recherché le soutien des paysans pauvres et des ouvriers, le régime s'était appuyé sur une bureaucratie d'état qui s'affirmait tandis qu'une récente bourgeoisie d'affaires bénéficiait de l'essor industriel. D'autre part, les minorités non sunnites, spécialement les Alaouites, avaient réussi à s'imposer dans le haut commandement militaire, reléguant les officiers sunnites dans les grades subalternes d'une armée considérée comme plus "politicienne" que professionnelle et jugée responsable de la défaite de 1967. Les années du "néo-baasisme" (1967-1970) avaient 
accentué ces recompositions et exacerbé les tensions en prônant un "socialisme scientifique" que les manifestants de 1973 dénonçaient comme "athée". Enfin, le "mouvement rectificatif" consécutif au coup d'état de Hafez Al-Asad en 1970, s'était traduit par une ouverture (infitâh), qui était avant tout économique, mais très peu politique : le régime baasiste avait évolué vers une formule présidentielle au détriment de la précédente formule collégiale, et si le parti avait été distingué de l'état, le second était de plus en plus identifié à la minorité alaouite contrôlant les postes stratégiques de l'armée. Cette ouverture avait en fait été ressentie, spécialement parmi les Sunnites, comme une négation des espoirs entrevus par la société civile ${ }^{12}$ Une nouvelle génération de Frères musulmans succédait à ceux des années 50, proches des nationalistes arabes. En dénonçant l'appropriation du régime syrien par les Alaouites, ils annonçaient les conflits particulièrement violents des années 1979-1982, dont ils seront les premières victimes lors de l'écrasement de la révolte de Hama en février 1982.

6 1986: les émeutes du Caire La soudaineté et l'imprévisibilité de ces émeutes, l'incertitude des réactions en égypte même, et la difficulté de les interpréter à la lumière de Faits plus anciens de l'histoire égyptienne ont fait de ces émeutes un événement atypique; du moins si l'on en cherche les "causes" directes. En revanche l'abondance de commentaires au cours de cette période en égypte illustre assez bien, par l'unanimisme momentané de réaction, la profondeur du trouble causé ${ }^{13}$ Rappelons les faits: suite à une "rumeur" selon laquelle les appelés des forces de la Sécurité centrale $^{14}$, ayant terminé leur service légal de trois ans, resteraient incorporés une année supplémentaire, ces derniers se mutinèrent dans les garnisons de la périphérie du Caire et se livrèrent à des actes de pillage et à l'incendie d'hôtels de tourisme et de cabarets situés dans la région des Pyramides. La rébellion fut réduite après deux jours d'affrontements entre ces unités et l'armée, et le couvre-feu maintenu dans toute la région du Grand-Caire pendant un peu plus de deux semaines. Quelle que soit l'explication avancée, en l'absence de véritable information un consensus apparut pour voir dans ces émeutes la manipulation d'appelés d'origine rurale, prêts à se révolter contre les conditions particulièrement sévères de leur service militaire. L'opposition emboîte alors le pas au président Mubarak qui dénonce dès le 26 février l'action téléguidée d'une "minorité délinquante", bien que tous n'y voient pas la même main. Si les commentaires sont allusifs pour évoquer le poids excessif pris par cette force de sécurité aux dépens de l'armée et sous-entendre les rôles ambigus du maréchal Abou Ghazala, ministre de la Défense et du ministre de l'Intérieur (qui sera contraint de démissionner), ils se font plus précis pour dénoncer le rôle joué à cette occasion par les islamistes du Jihâd ${ }^{15}$, ou par des "marxistes influencés par l'étranger Syrie et Libye) et résolus à nuire à l'égypte. Ce réflexe de solidarité nationale passé, l'événement paraît de nature à cristalliser des positions politiques variées, influencées par une instabilité chronique depuis l'assassinat de Sadate. C'est dans ces réactions que l'on peut déceler des perceptions nouvelles quant à la pertinence politique des mouvements de rébellion égyptiens. Hormis quelques analyses originales et isolées d'intellectuels évoquant le "syndrome libanais"16 ou les stratégies de violence propres à la vie politique égyptienne depuis la guerre entre Nasser et les Frères musulmans ${ }^{17}$ la majorité des réactions parues par voie de presse peuvent se regrouper en trois types principaux. La majorité présidentielle ne veut voir dans ces faits qu'une agitation conjoncturelle "antiégyptienne" qui doit être réprimée par la loi. La gauche nassérienne, tout en condamnant la rébellion, considère qu'elle est l'expression de trois facteurs de 
déséquilibre profond : la crise économique et sociale dont témoignent des mutins issus de campagnes durement touchées; la permanence de la question palestinienne que les accords de Camp David n'ont nullement atténuée ${ }^{18} ; l^{\prime}$ absence d'une démocratie réelle malgré les ouvertures parlementaires de 1984, consécutives à l'infitâh. Si la gauche nassérienne se déclare aussi préoccupée par l'aggravation des incidents interconfessionnels au cours des années précédentes, c'est surtout un dernier courant qui met l'accent sur les facteurs "confessionnels" de désagrégation sociale et sur la signification de l'événement au regard de la culture islamique. On insiste notamment sur les cibles visées par les mutins : les hôtels touristiques, signes visibles de l'intrusion du luxe occidental et les cabarets de l'avenue des Pyramides, "lieux de débauche, de consommation d'alcool et de jeux de hasard" que du reste ne dédaignent pas les ressortissants saoudiens. Pour le journal Al-Sha'ab, organe du Parti du travail, l'existence de ces lieux "où l'on commerce l'illicite" justifie la revendication d'une complète application de la sharî'a ${ }^{19}$. Fort différent de cette approche teintée de populisme et réactive à la peur causée par la soudaineté des faits (les médias ont favorisé cette réaction) est cependant l'écho donné par les grands oulémas égyptiens à l'affaire; ils laissent percer leur inquiétude devant des agissements dans lesquels ils voient les signes d'une crise des valeurs, exploitée par ces "usurpateurs" que sont les islamistes. Si la justice doit être distribuée au nom de la sharî'a, elle doit l'être contre les fauteurs de troubles qui servent des desseins illicites. C'est ainsi que les mutins sont classés comme des producteurs de fitna (sédition contre la umma) et doivent subir les rigueurs d'une justice islamique qui ne devrait pas se confondre avec le droit pénal égyptien. Le shaykh Shaarawi en particulier taxe les mutins de brigandage (hirâba) ${ }^{20}$, passible en droit islamique de la peine de mort, et le shaykh Al-Azhar, Ali Gadd al-Haqq, appelle sur eux l'application des hudûd (châtiments légaux islamiques) ${ }^{21}$. Les événements égyptiens de 1986 ont pu être perçus comme la péripétie dramatique d'une recomposition de l'équilibre des forces armées au sein de l'état égyptien, à laquelle assistait une population quasi-indifférente et dont un nombre restreint de citoyens particulièrement démunis ont été les acteurs et les victimes. Ils témoignent une fois de plus de l'essoufflement d'un pouvoir politique qu'une ouverture tardive n'a pas su empêcher. L'esquisse d'un pluralisme à l'Assemblée du peuple en 1984 a fourni une tribune aux courants islamistes modérés, sans permettre au gouvernement de freiner le processus de délégitimation qui l'atteint et de réduire l'impasse où l'a conduit l'absence d'une réelle démocratisation. L'invocation de la sharî'a islamique pour fonder une régulation meilleure des rapports sociaux est désormais faite de façon quasi-rituelle par des acteurs qui, indépendamment de leurs options politiques, se déclarent persuadés que la "solution" est désormais d'ordre beaucoup plus culturel que social ou économique ${ }^{22}$.

7 L'Algérie depuis 1988 Dans un tout autre contexte, l'Algérie de 1988 est devenue le point de mire, car la capacité qu'ont eu des groupes militants relativement réduits se réclamant de l'islam politique d'interpréter les aspirations d'un nombre important de citoyens, a fait éclater au grand jour la possibilité - sinon la pertinence - d'une solution "islamique" exprimée dans le slogan : "L'islam est la solution" (alislâm huuwwa al-hall). On peut voir dans le soulèvement algérien de 1988 la réédition des "émeutes FMI" de 1984, mais accrue d'une potentialité contestataire captée par ces nouveaux représentants de la société civile que sont désormais les islamistes. Provoquées par la suppression de l'allocation de devises aux Algériens se rendant à l'étranger, la raréfaction des produits de première nécessité et une hausse généralisée des prix, les 
manifestations qui ont lieu à partir du 2 octobre 1988 sont canalisées par une manifestation islamiste au sortir de la prière du vendredi ? octobre. Ali Belhadj peut se poser en médiateur entre l'armée et les manifestants. Le 10, le président Chadli Bendjedid sanctionne cette situation en recevant une délégation islamiste composée de Belhadj, Sahnoun, Nahnah et Madani qui lui remettent un "cahier de doléances". Le FIS, constitué peu après, en mars 1989, s'imposera comme le porte-parole quasi-exclusif d'une contestation qui s'exprimera non seulement dans la rue, mais dans la conquête de positions électorales aux élections communales de juin 1990, puis législatives du 26 décembre 1991.

8 Si le FIS s'est présenté explicitement comme le dépositaire d'un modèle politique basé sur une interprétation de la culture islamique, il a bénéficié d'une mobilisation plus ancienne sur le thème de la réislamisation de la société. La situation particulière de l'Algérie, pays arabe francophone et comprenant une importante minorité berbérophone, a rendu moins visible l'ascension de sa composante islamiste, en comparaison notamment avec la Tunisie. Mais pratiquement dès l'indépendance, des thèmes dont certains sont repris de l'ancienne Association des oulémas fondée par Abd al-Hamid Ibn Badis, ont défini des "itinéraires de mobilisation" spécifiques ${ }^{23}:$ statut de l'enseignement religieux, rétabli en 1964 par Ben Bella sous la pression de l'association Al-Qiyam nouvellement créée, débats à partir de 1975 autour du droit de la famille et surtout statut de nouveaux diplômés en arabe, qui se trouvent frustrés de ne pouvoir bénéficier pleinement des débouchés d'une formation universitaire dévaluée par rapport aux filières effectuées en français. Ces éléments d'identification nourrissent des contre modèles dans un contexte où la démographie et l'endettement croissant contribuent à grossir le nombre des mécontents et à radicaliser une opposition à laquelle le FLN n'a fait de concessions d'ouverture que quand il y fut forcé par la rue.

Stratégies nationales de tension et contexte international Modèles contestataires dans le nouveau contexte international L'islamisme algérien est le plus récent des islamismes arabes. Il propose, à partir du Maghreb, un modèle contestataire relayant celui mis à l'épreuve par la révolution iranienne. Son développement au cours des deux dernières décennies et surtout de la dernière, a tenu compte d'expériences antérieures, comme l'attestent ses nombreuses références au mouvement des Frères musulmans et à sa variante qutbiste ${ }^{24}$. Les trois cas que nous avons présenté témoignent, parmi d'autres, d'une maturation de la réflexion sur les conditions de réalisation d'un islam politique transnational. La crise syrienne de 1973 révèle à la société civile ses capacités "islamiques" de contestation de gouvernements de plus en plus personnalisés et incapables de comprendre qu'ils ne répondent pas à ses attentes d'ouverture réelle. L'égypte des débuts de l'infitâh a largement stigmatisé le "terrorisme" des Gamâ'ât islamiques. Dix ans plus tard, en 1986, les principales formations politiques ont en bonne part intériorisé la doctrine islamiste. Non pas tant les contenus d'un état califal que l'idéologie ne parvient guère à décrire ${ }^{25}$ que la simple conviction dans l'efficacité d'un langage politique "islamisé" qui répond aux attentes d'une partie importante de la population. Des militants organisés ont su répondre à ces attentes et accéder à la direction de la contestation. Cette dynamique ne doit cependant pas tout à la contestation islamiste. On pourra citer, dans le cas du Maghreb, des mobilisations externes au mouvement islamiste, voire des contre-mobilisations qui ne semblent pas gêner la propagande des radicaux et peuvent même lui profiter. C'est le cas au Maroc où une importante manifestation de soutien à l'Irak, réunie le 2 février 1991 par les syndicats et les partis d'opposition, fut tolérée par le gouvernement qui venait 
d'affronter durant tout le mois de décembre 1990, l'organisation d'une grève générale accompagnée le 14, de violentes émeutes de jeunes issus de quartiers pauvres, à Kenitra, Tanger et surtout Fès; manifestants prompts à se radicaliser et à s'identifier aux "déshérités" que défendent les islamistes ${ }^{26}$. En 1989 et 1990 en Algérie, différentes marches laïcistes, organisées au nom du droit des femmes, de l'identité amazigh (berbère), au nom des "patriotes" (manifestations FLN) ou "pour la démocratie et la tolérance" n'ont pu empêcher des contremarches plus imposantes organisées par les islamistes et de plus en plus canalisées par le FIS. Le succès de cette surenchère démontre l'échec d'un système politique associé au FLN et que les élections législatives du 26 décembre 1991 viennent de rendre plus éclatant. En dépit d'une forte abstention des électeurs, la nouvelle poussée du FIS a su profiter d'un vote-sanction du FLN, d'un "vote de vengeance", pour reprendre l'expression de commentateurs algériens. La guerre du Golfe n'est certes pas étrangère à cette recomposition. Dans un premier temps, le FIS a été déstabilisé par ses engagements antérieurs vis-à-vis de l'Arabie Saoudite, que celle-ci, ainsi que le gouvernement algérien, ne se sont pas privés de rappeler. Mais après avoir condamné l'invasion irakienne du Koweït, il s'est senti assez fort pour dénoncer l'occupation des lieux saints par des armées infidèles et "l'inaction" du FLN devant ce qui était vécu en Algérie comme l'agression d'un peuple frère. Ces difficultés momentanées expliquent une faible capacité mobilisatrice initiale (moins de 100.000 personnes), lors d'une manifestation prévue "imposante" le 31 janvier 1991, mais vite compensée par des manifestations ultérieures plus massives. L'échec de Saddam Hussein sur le terrain ne signifie pas celui des idées dont il se présentait comme le dernier défenseur, en réunissant dans une contestation globale, la défense des peuples irakien et palestinien, et la réaffirmation d'une identité arabe et islamique défiée par les armées de la coalition ${ }^{27}$.

La contestation islamiste en Algérie A cette nouvelle étape de la contestation islamiste, comment s'exerce la tension jusqu'à présent entretenue par des organisations désignées et combattues comme "extrémistes" ou "terroristes" par les états (en égypte, en Tunisie ou au Maroc) ? En Algérie, après avoir remporté les élections municipales et de wilaya de juin 1990 et s'être assuré une majorité potentielle au premier tour des élections législatives de décembre 1991, le FIS était en passe de recevoir une consécration institutionnelle. Or, on le sait, l'armée algérienne a mis un coup d'arrêt le 12 janvier 1992 à cette progression. Les cadres les plus importants du Front ayant été emprisonnés et son organisation malmenée, des questions se posent quant à sa capacité de redéfinir une stratégie contestataire adaptée à la situation, compte tenu de la frustration de militants et de sympathisants privés d'une victoire qui était tenue pour acquise, dans un cadre légaliste. La situation algérienne ayant eu un écho indéniable dans le monde arabe, et spécialement au Machreq, il faut rappeler ce qui fait sa spécificité interne, et au plan international examiner l'hypothèse d'une recomposition de mouvements islamistes affrontés à la répression des états. Au plan interne, on observera deux choses. En premier lieu, le FIS a élaboré une théorie de la "violence légitime", non pas dans son programme électoral, légaliste, mais dans les discours de ses dirigeants. Du reste, diverses actions publiques de militants islamistes ont "rappelé" l'obligation coranique du "commandement du bien et de l'interdiction du mal". Cette théorie de la violence légitime ou de la contre-violence s'articule de la façon suivante ${ }^{28}$ : la société algérienne subit l'agression d'un mode de vie importé d'Occident et de façon très concrète, les militants islamistes ont été les victimes de la répression du gouvernement. II s'agit d'une campagne orchestrée par les médias, une partie des 
intellectuels et des oulémas, les partis laïcistes et les appareils de l'état; ses objectifs sont à court terme de dresser l'opinion nationale et internationale contre le mouvement et à long terme, d'affaiblir la conscience religieuse de la société et "l'esprit de djihâd" chez les jeunes. Face à cette violence légale, une contre-violenee, légitime, s'impose. En second lieu, cette logique réactive, influencée par des écrits élaborés au Machreq, peut-elle faire place ultérieurement à une dynamique constructive, en posant comme hypothèse que le mouvement ne se laisserait pas enfermer dans un immobilisme doctrinal, aujourd'hui de mise ? La question posée ${ }^{29}$ est celle de la capacité de ses intellectuels à produire une interprétation nouvelle (ijtihâd) de la loi islamique pour tenir compte autant des contraintes économiques et sociales que des revendications exprimées depuis 1988 en faveur d'un système politique ouvert. Le récent coup d'état militaire a sans doute rendu cette question caduque pour un temps, mais il n'a pas pour autant supprimé sa pertinence pour les années à venir. Au plan international, une stratégie délibérée de la tension peut apparaître "opportune", mais elle est incertaine. Après la guerre du Golfe, l'éventualité d'une conjoncture favorable au rassemblement de forces islamistes autour d'états tels que l'Iran ou le Soudan ne supprime pas la difficulté de concevoir une solidarité réelle et durable entre des mouvements populaires et des états ${ }^{30}$. En revanche, l'éventualité de stratégies communes à divers mouvements islamistes dépend, dans la durée, outre leurs conditions nationales d'expression, de leur capacité à surmonter des divergences doctrinales souvent tenaces, comme c'est le cas, on le sait, entre chiites et sunnites. Et ce, d'autant plus que l'idéologie khomeyniste n'a que lentement pénétré le monde arabe sunnite jusqu'à ces dernières années, en particulier parce que la littérature politique iranienne fut peu traduite en arabe, au moins pendant les premières années de la révolution de $1979^{31}$. Cela n'a pas exclu un évident impact de la révolution khomeyniste. Les militants algériens reconnaissent aujourd'hui l'antériorité dans la structuration d'un champ politique islamique(31), des références à l'idéologie des islamistes iraniens. Ces processus sont néanmoins incertains. Tout d'abord des regroupements "anti-impérialistes" ont déjà existé dans le passé avec des objectifs plus ou moins ambitieux, qu'il s'agisse de la Ligue arabe, des expériences limitées d'unité arabe ou de l'Organisation de la Conférence islamique et ces expériences se sont heurtées à la difficulté de définir un projet commun universalisable. Même en faisant abstraction de divergences idéologiques qui sans doute pèsent peu face à des opportunités politiques de regroupement contre un "impérialisme mondial" désigné comme la cible principale, il n'est pas sûr que des états puissent s'associer durablement avec des mouvements politiques parfois "légalisés" par les états, mais non véritablement institutionnalisés. En prenant fait et cause pour les militants algériens victimes de l'intervention militaire, les dirigeants iraniens tiennent compte des nouveaux équilibres issus de la guerre du Golfe et de leur rivalité avec l'Arabie Saoudite. Quant aux islamistes maghrébins et surtout algériens, même s'ils n'ont sans doute rien perdu de leur combativité en dépit de leurs récents déboires, ils savent qu'ils doivent composer avec ces équilibres.

11 * Chargé de recherches à l'IRENAM (CNRS, Aix en Provence) 


\section{NOTES}

1. Cf. en 1955 le pacte de Bagdad.

2. Que des commentateurs occidentaux se soient employés pendant la guerre du Golfe à en limiter la portée en dénonçant le "suivisme" de l'OLP à l'égard des dirigeants de Bagdad ne réduit en rien le fait que le "lien" de la question palestinienne avec les événements du Golfe était actif dans les représentations politiques du monde arabe.

3. Par exemple, à l'époque de la RAU (1958-1961), les Frères musulmans syriens ont mis au point une doctrine du "socialisme islamique" inspirée de Rachid Ridha, qui s'accommode alors sans difficulté du nassérisme.

4. Cf. par exemple John Waterbury, "La légitimation du pouvoir au Maghreb : tradition, protestation et répression", in Développements politiques au Maghreb, (ouvr. coll.), Paris, CNRS, 1979.

5. Terme coranique dont se sont abondamment servis depuis la révolution iranienne, les mouvements islamistes. Sur son utilisation par Saddam Hussein dans le contexte de la guerre du Golfe, voir Bertrand Badie, "La guerre contestataire", in REMM, 1990, Crise du Golfe. La logique des chercheurs, pp. 54-56.

6. Sur le rôle de l'Islam dans les mobilisations politiques des sociétés arabes du XIXème siècle, voir Edmond Burke III et Ira M. Lapidus, Islam, Politics and Social Movements, London, I.B. Tauris, 1988.

7. . Comme le dit pour l'Egypte, Muhammad Nour Farahat in Al-Hilâl, avril 1986.

8. Cf. Ghassam Salamé, "Sur les causalités d'un manque. Pourquoi le monde arabe n'estil donc pas démocratique", Revue française de science politique, 3-1991.

9. . Comme le dit pour l'Egypte, Muhammad Nour Farahat in Al-Hilâl, avril 1986.

10. Cf. B. Botiveau, Sharî'a islamique et droit positif dans le Moyen-Orient contemporain, Thèse, Aix-en-Provence, 1989, pp. 223-229.

11. Par référence de la formule coranique : "Dirige-nous dans la Voie Droite (al-sirât almustaqîm), Sourate liminaire, 6 .

12. Les Frères musulmans ne sont pas alors les seuls militants de la contestation. En 1973, des militants nassériens, il est vrai en perte de vitesse, se sont joints à eux dans les émeutes dont il est question.

13. Sur ces événements et leur interprétation en Egypte au cours des premiers mois de 1986, voir le dossier de presse constitué par la Revue de la presse égyptienne, Le Caire, CEDEJ, $n^{\circ}$ 22-1986, pp. 135-206.

14. Al-amn al-markazi : force de la police dépendant du ministère de l'Intérieur, créée par Nasser après la défaite de 1967 et dont l'importance s'est considérablement accrue pour atteindre une mobilisation de 300000 hommes.

15. Qui apparaît lors de l'attaque du bagne de Tura, symbole de la répression nassérienne contre les Frères musulmans, et où sont détenus des membres de l'Organisation dont certains ayant été impliqués dans d'assassinat de Sadate.

16. Anis Mansour dans Al Ahrâm du 28.02.1986.

17. Kamal Abu Al-Magd ou Muhammad Nour Farahat rians Al-Hilâl d'avril 1986.

18. On rappelle alors abondamment les événements révélateurs de l'année 1985 ; en particulier, attentats contre des diplomates israéliens, affaire Suleyman Khater.

19. Avis également de l'hebdomadaire Al-Nûr du 5.03. 1986.

20. Selon Al-Mujahid de mars $1986 .$. 
21. AlAhrâm du 28.02.1986.

22. L'islamisation généralisée des idiomes politiques est l'un des traits caractéristiques de ces années, cf. Alain Roussillon, "Entre Al-Jihad et Al-Rayyan : phénoménologie de l'islamisme algérien", Maghreb-Machreq, n 127, 1990.

23. Cf. François Burgat, L'islamisme au Maghreb, Paris, Karthala, 1988, pp. 152 et s.

24. Voir les textes du mouvement réunis in M. Al-Ahnaf B. Botiveau, F Frégosi, L'Algérie, par ses islamistes, Paris, Karthala, 1991.

25. Ibid., Ch. II, "la politique selon le Fis".

26. Selon l'opposition marocaine, il y eut des dizaines de morts et de blessés durant ces émeutes, le jour même de la grève générale. Quant aux manifestations de début février, elles ont été largement suivies, réunissant notamment 5011 000personnes à Rabat 27. Cf. l'opinion de l'Ecrivain algérien Rachid Mirnouni, "De la chute de Saddam au regain islamiste" in Libération du 29 juillet 1991.

28. voir en particulier un prêche de Ali Belhadj, L'Algérie par ses islamistes, op. cit., pp. 132 et $\mathrm{s}$.

29. François Burgat, "Eléments de prospective sur la mobilisation islamiste en Méditerranée", in L'Etudes politiques du monde arabe, Le Caire, Dossiers du CEDFJ 1991, pp. 307-314.

30. Cf. Bertrand Badie, art. cit.

31. Cf. Emmanuel Sivan, "Sunni Radicalism in the Middle East and the Iranian Revolution", International Journal of Midlle East Studies, 21 (1989), pp. 1-30. 31. Contestant l'attirance de certains prédicateurs algériens pour les thèses chiites, Ali Belhadj n'en concède pas moins que, l'état iranien islamique étant en formation, "il fallait se taire bien qu'on sût que c'était de mauvaises croyances (i.e. les croyances chiites), pour ne pas faire le jeu de l'impérialisme mondial", cf. L'Algérie par ses islamistes, op. cit., pp. 70-71.

\section{INDEX}

Mots-clés : résistances politiques, mobilisation Index géographique : Maghreb, Moyen-Orient 